\title{
Compact low-birefringence polarization beam splitter using vertical-dual-slot waveguides in silicon carbide integrated platforms
}

Shi, Xiaodong; Zhang, JingJing; Fan, Weichen; Lu, Yaoqin; Peng, Nianhua; Rottwitt, Karsten; Ou, Haiyan

Published in:

Photonics Research

Link to article, DOI:

10.1364/PRJ.443543

Publication date:

2021

Document Version

Peer reviewed version

Link back to DTU Orbit

Citation (APA):

Shi, X., Zhang, J., Fan, W., Lu, Y., Peng, N., Rottwitt, K., \& Ou, H. (2021). Compact low-birefringence polarization beam splitter using vertical-dual-slot waveguides in silicon carbide integrated platforms. Photonics Research, 10(1), A8-A13. https://doi.org/10.1364/PRJ.443543

\section{General rights}

Copyright and moral rights for the publications made accessible in the public portal are retained by the authors and/or other copyright owners and it is a condition of accessing publications that users recognise and abide by the legal requirements associated with these rights.

- Users may download and print one copy of any publication from the public portal for the purpose of private study or research.

- You may not further distribute the material or use it for any profit-making activity or commercial gain

- You may freely distribute the URL identifying the publication in the public portal 


\title{
Compact low-birefringence polarization beam splitter using vertical-dual-slot waveguides in silicon carbide integrated platforms
}

\author{
Xiaodong Shi ${ }^{1}$, Jinguing Zhang ${ }^{2}$, Weichen Fan ${ }^{1}$, Yaoqin LU ${ }^{1}$, Nianhua Peng ${ }^{3}$, Karsten \\ ROTTWITT ${ }^{1}$, AND HAIYAN OU ${ }^{1, *}$ \\ ${ }^{1}$ DTU Fotonik, Technical University of Denmark, DK-2800 Lyngby, Denmark \\ ${ }^{2}$ Institute of Mirco/Nano Optoelectronic and Terahertz Technology, Jiang Su University, Jiangsu, 210010, China \\ ${ }^{3}$ Surrey lon Beam Centre, Surrey University, Guildford, GU2 7XH, UK \\ *Corresponding author: haou@fotonik.dtu.dk \\ Compiled November 18, 2021
}

\begin{abstract}
The polarization beam splitter is a key component for polarization manipulation in photonic integrated circuits, but it is challenging to design for low-refractive-index optical materials, due to the low birefringence of the waveguides. We propose a novel compact vertical-dual-slot waveguides based coupling scheme for silicon carbide, enabling efficient low-birefringence polarization splitting by extensively modulating the transverse-magnetic mode distribution. We numerically and experimentally demonstrate the device in the $4 \mathrm{H}$-silicon carbide-on-insulator integrated platform, with a small footprint of $2.2 \times 15 \mu \mathrm{m}^{2}$. The device, easy to fabricate via single lithography process as other components on the chip, exhibits low insertion loss of $<0.71 \mathrm{~dB}$ and $<0.51 \mathrm{~dB}$ for the transverseelectric and transverse-magnetic polarized light, respectively, and polarization extinction ratio of $>13 \mathrm{~dB}$, over $80 \mathbf{~ n m}$ wavelength range. () 2021 Optical Society of America
\end{abstract}

http://dx.doi.org/10.1364/ao.XX.XXXXXX

\section{INTRODUCTION}

Silicon carbide $(\mathrm{SiC})$ has attracted great interests in both classical and quantum integrated photonics during the past decade, thanks to its wide bandgap, excellent second- and third-order nonlinear properties, and a variety of intrinsic color centers $[1,2]$. These characteristics have been employed to achieve miscellaneous applications, including optical parametric oscillation, frequency comb generation, electro-optic modulation, and single photon sources, making possible monolithic $\mathrm{SiC}$ quantum integrated circuits [3-8]. Among more than 200 crystalline $\mathrm{SiC}$ polytypes, $4 \mathrm{H}-\mathrm{SiC}$ is one of the most widely used polytypes for integrated photonics, due to its high crystal quality and low optical loss [5]. As a uniaxial crystal, $4 \mathrm{H}-\mathrm{SiC}$ exhibits orientation-dependent and polarization-dependent optical properties, including linear refractive index and nonlinear refractive index. It means that the transverse-electric (TE) and transverse-magnetic (TM) polarized light, propagating along the $4 \mathrm{H}-\mathrm{SiC}$ waveguides, typically has different properties, such as polarization-dependent loss, polarization-dependent dispersion, and polarization-dependent nonlinear strength [9]. Thus, polarization control and management in the $4 \mathrm{H}-\mathrm{SiC}$ integrated platform are highly important, and are worthy exploring.

The polarization beam splitter (PBS), a key component for polarization manipulation, enables two orthogonal mode separation and provides polarization diversity in photonic integrated circuits, which can also be applied for the polarization division multiplexing and the polarization entanglement quantum systems [10, 11]. Many different types of PBSs have been studied in silicon-on-insulator integrated platforms, for example, directional couplers, multimode interferometers, gratings, and plasmonic waveguides [12-17]. Although silicon $(n=3.5$ at $1550 \mathrm{~nm})$ does not have material-based birefringence, high geometric birefringence in silicon waveguides makes easy polarization beam splitting, as the phase-matching condition cannot be satisfied by two orthogonal modes simultaneously due to the large effective refractive index contrast. Waveguides, made of optical materials with lower refractive index, such as $\mathrm{SiC}(n=2.6)$, silicon nitride $(\mathrm{SiN})(n=2.0)$ and lithium niobate $\left(\mathrm{LiNbO}_{3}\right)(n=2.2)$ at $1550 \mathrm{~nm}$, usually require larger geometry to confine the light, and have lower birefringence, compared to silicon waveguides. For example, the effective refractive index of the fundamental TE and TM modes in a typical standard single-mode silicon waveguide with a dimension of $220 \times 500 \mathrm{~nm}^{2}$ at $1550 \mathrm{~nm}[13,14]$, is 2.39 and 1.59 , respectively, but becomes 1.79 and 1.63 , respectively, in a typical single-mode $4 \mathrm{H}-\mathrm{SiC}$ waveguide with a dimension of $350 \times 560 \mathrm{~nm}^{2}$ [1], which shows much lower birefringence. Therefore, it is a challenge to make compact and efficient PBSs in the relatively low-refractive-index optical materials based integrated platforms [18].

Several methods have been proposed and demonstrated in $\mathrm{LiNbO}_{3}$ and SiN integrated platforms. Multimode interferometers and MachZehnder interferometers are the most promising approaches to make 
good-performance PBSs with low-refractive-index optical materials [18-21]. Especially, high extinction ratio $(>20 \mathrm{~dB})$ can be achieved by tailoring the phase shifting region. However, such devices usually have large footprint of thousands of square micrometers, which is not good for compactness and high-density integration. Metamaterial has been proposed to design anisotropic medium with artificially generated strong birefringence, making possible efficient PBSs, but complex structures with high-precision fabrication are needed [22]. Threedimensional multiple-layer structures and assisted amorphous silicon waveguides offer capability to make efficient and broadband PBS, but introducing extra layers or materials requires multiple lithography and complicated fabrication processing [23, 24]. Cascaded asymmetric directional couplers, that convert fundamental TM modes to first-order modes, enable high-performance PBSs, fabricated by a standard singlestep lithography process, and the device length is as short as $112 \mu \mathrm{m}$ [25]. Generally, it remains a demanding challenge to achieve ultracompact and efficient low-birefringence PBSs, conformed to simple fabrication processes, and there is also a lack of PBSs in SiC integrated platforms.

In this work, we design a novel vertical-dual-slot waveguide coupler based PBS for low-birefringence waveguides. Vertical-dual-slot waveguides have been used for group-velocity dispersion tailoring and efficient electro-optic modulation by enhancing the optical and electric field overlap [26, 27]. Here, we use this structure to adjust the mode distribution for polarization splitting. It is found that the TM mode is particularly sensitive to the vertical-dual-slot waveguide in regard of the mode distribution. By adjusting the TM mode distribution, the coupling strength between the two waveguides is strongly modulated. The simple structure is also compatible with the standard single-step lithography fabrication process. We also experimentally demonstrate the $\mathrm{PBS}$ in the $4 \mathrm{H}-\mathrm{SiC}$-on-insulator (SiCOI) integrated platform. The device exhibits low insertion loss of $<0.71 \mathrm{~dB}$ and $<0.51 \mathrm{~dB}$ for the TE and TM polarized light, respectively, and polarization extinction ratio of $>13 \mathrm{~dB}$ from $1540 \mathrm{~nm}$ to $1620 \mathrm{~nm}$, within a very small coupling region of $2.2 \times 15 \mu \mathrm{m}^{2}$. To our best knowledge, this is the first demonstration of a PBS in SiC integrated platforms.

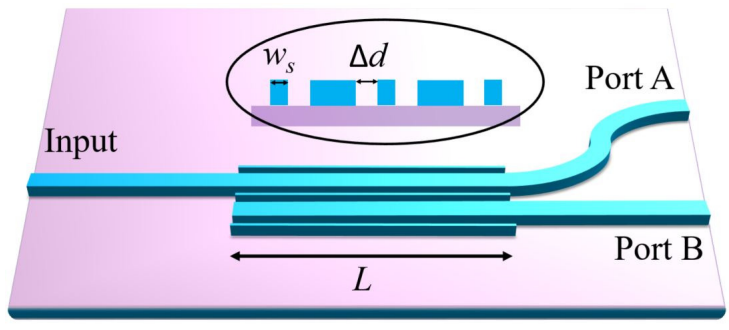

Fig. 1. Schematic of the proposed PBS with vertical-dual-slot waveguides.

\section{DESIGN AND SIMULATION}

Figure 1 shows the schematic of the proposed vertical-dual-slot waveguide based PBS, and the inset shows the cross section of the coupling region of the PBS, which consists of two cores, four air slots and three strips. The central core is a single-mode $4 \mathrm{H}-\mathrm{SiC}$ waveguide, and has a dimension of $400 \times 600 \mathrm{~nm}^{2}$. The slot has a width of $\Delta d$, and the side strip has a width of $w_{s}$ with the same height as the central core. The coupling region, with a length of $L$, is composed of two parallel vertical-dual-slot waveguides, sharing the same side strip in between. Light is injected into the single-mode waveguide from the left input port. After the PBS, the TE polarized light remains in the upper waveguide and goes out from Port A, while the TM polarized light couples into the other waveguide and goes out from Port B.
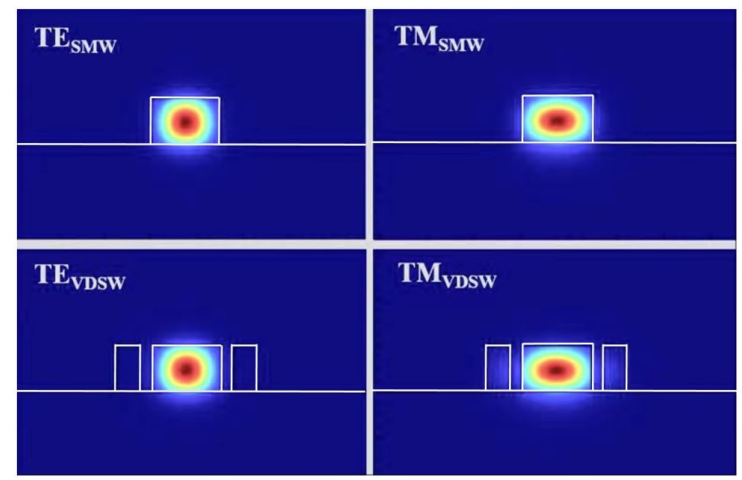

Fig. 2. Energy density profiles of TE and TM modes in the 4H-SiC single-mode waveguides (SMW) and the vertical-dual-slot waveguides (VDSW). In the simulation, the SMW has a dimension of $400 \times 600 \mathrm{~nm}^{2}$, the VDSW has a strip width of $200 \mathrm{~nm}$ and a slot width of $100 \mathrm{~nm}$, the refractive index of the $\mathrm{SiC}$ is 2.56 and 2.60, for the TE and TM mode, respectively, and the refractive index of the $\mathrm{SiO}_{2}$ is 1.44 .

We design and simulate the PBS using Lumerical MODE and FDTD softwares. The energy density distribution of the TE and TM modes in the $4 \mathrm{H}-\mathrm{SiC}$ single-mode waveguides and the vertical-dual-slot waveguides is shown in Fig.2. It is seen that both TE and TM modes are well confined in the single-mode waveguides, and have similar effective refractive index of 1.90 and 1.78 , respectively. The effective mode area of the TE and TM modes is $0.38 \mu \mathrm{m}^{2}$ and $0.47 \mu \mathrm{m}^{2}$, respectively. However, when employing the vertical-dual-slot structure, for example, with a dimension of $w_{s}=200 \mathrm{~nm}$ and $\Delta d=100 \mathrm{~nm}$, the TM mode distribution can be largely changed, but the TE mode distribution almost remains unchanged. The effective refractive index of the TE and TM modes is 1.92 and 1.82, respectively, almost the same as those in the single-mode waveguide, but the effective mode area of TE and TM modes becomes $0.37 \mu \mathrm{m}^{2}$ and $0.57 \mu \mathrm{m}^{2}$, respectively. The TM mode distribution is much more strongly affected by the vertical-dualslot structure, especially with an apparent lateral expansion, compared to the TE mode. The strong polarization-dependent mode distribution modulation provides the opportunity to realize compact and efficient polarization beam splitting with the proposed structure.

We optimize three parameters in the vertical-dual-slot waveguide based PBS, including the side strip width, the slot width, and the coupling length. The minimal side strip width and the slot width are limited by the minimal feature size of the fabrication process, which is about $100 \mathrm{~nm}$. The coupling length of the TM mode is estimated to be $17.3 \mu \mathrm{m}$, according to the coupling theory of the directional couplers, given by $L=\frac{\lambda}{2\left(n_{\text {even }}-n_{\text {odd }}\right)}$, where $\lambda$ is the wavelength of the lightwave, $n_{\text {even }}=1.837$ and $n_{\text {odd }}=1.792$ are its effective refractive index of the even symmetric mode and the odd symmetric mode, respectively [28]. While the coupling length of the TE mode is $97 \mu \mathrm{m}$, much longer than that of the TM mode. The transmission efficiency versus the coupling length is plotted in Fig.3(a), with fixed side strip width and slot width of $200 \mathrm{~nm}$ and $100 \mathrm{~nm}$, respectively. The transmission of TE polarized light decreases with the coupling length. The highest transmission of TM polarized light is between $17 \mu \mathrm{m}$ and $18 \mu \mathrm{m}$, well matching the calculation. However, the performance of the TE polarized light is not as good as that of the TM polarized light propagating through the PBS within this range, but has lower coupling efficiency and higher cross talk. Thus, we choose the crossing point from Fig.3(a), which is at $15 \mu \mathrm{m}$, so that both TE and TM polarized light can have high 


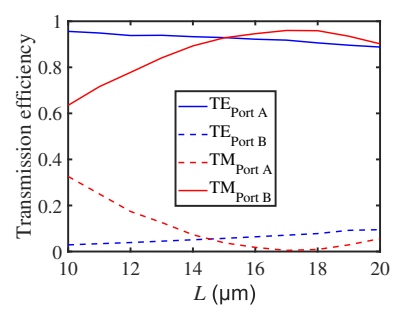

(a)

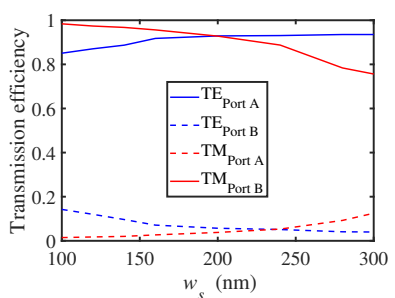

(b)

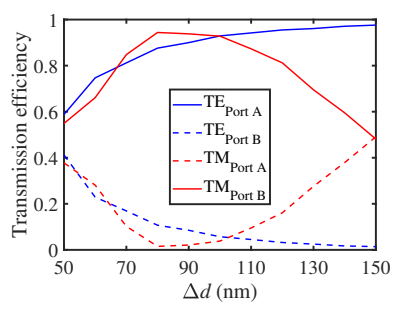

(c)

Fig. 3. The simulated results of the transmission efficiency as a function of (a) the coupling length with a side strip width of $200 \mathrm{~nm}$ and a slot width of $100 \mathrm{~nm}$, (b) the side strip width with a slot width of $100 \mathrm{~nm}$ and a coupling length of $15 \mu \mathrm{m}$, and (c) the slot width with a side strip width of $200 \mathrm{~nm}$ and a coupling length of $15 \mu \mathrm{m}$.

transmission efficiency and large polarization extinction ratio, given by $E R_{\mathrm{TE}}=10 \log _{10} \frac{T_{\mathrm{TE}_{\text {Port }} \mathrm{A}}}{T_{\mathrm{TE}_{\text {Port }} \mathrm{B}}}$ and $E R_{\mathrm{TM}}=10 \log _{10} \frac{T_{\mathrm{TM}_{\text {Port }} \mathrm{B}}}{T_{\mathrm{TM}_{\text {Port }} \mathrm{A}}}$, for TE and $\mathrm{TM}$ polarized light, respectively. Within the coupling length range from $14.5 \mu \mathrm{m}$ to $15.5 \mu \mathrm{m}$, both TE and TM polarized light has a high transmission efficiency of $>90 \%$, equivalent to an insertion loss of $<0.46 \mathrm{~dB}$, given by $I L_{\mathrm{TE}}=-10 \log _{10} T_{\mathrm{TE}} \mathrm{Port} \mathrm{A}_{\text {A }}$ and $I L_{\mathrm{TM}}=-10 \log _{10} T_{\mathrm{TM}}{ }_{\text {Port B }}$, for TE and TM polarized light, respectively, and a polarization extinction ratio of $>12 \mathrm{~dB}$. The transmission efficiency versus the side strip width is plotted in Fig.3(b), with fixed slot width and coupling length of $100 \mathrm{~nm}$ and $15 \mu \mathrm{m}$, respectively. The transmission efficiency of the TE polarized light increases with the side strip width, while that of the TM polarized light decreases. Narrow side strip width enables efficient TM polarized light coupling, but results in high crosstalk of the TE polarized light, due to the close distance between the two coupling central waveguides. Wide side strip width could suppress the TE polarized light coupling, but also weaken the TM polarized light coupling, in spite of strong TM mode distribution modulation. Within the side strip width range from $160 \mathrm{~nm}$ to $220 \mathrm{~nm}$, both TE and TM polarized light has a high transmission efficiency of $>90 \%$ and a polarization extinction ratio of $>12 \mathrm{~dB}$. Such wide side strip width range allows a high fabrication dimension tolerance. The transmission efficiency versus the slot width is plotted in Fig.3(c), with fixed side strip width and coupling length of $200 \mathrm{~nm}$ and $15 \mu \mathrm{m}$, respectively. It can be seen that the device is more sensitive to the slot width than the side strip width. The transmission efficiency of the TE polarized light increases with the increasing slot width, while that of the TM polarized light first increases and then decreases. Narrow slot width expands the TM mode intensively, and compresses the TE mode at the same time, but with the current coupling length, the TM polarized light does not have enough power coupling, and the close distance between the two coupling central waveguides results in high crosstalk. Wide slot width helps to increase the transmission efficiency of the TE polarized light, but also reduces the TM polarized light coupling, due to the weak TM mode distribution modulation and the long distance between the two coupling central waveguides. Within the slot distance range from 90 $\mathrm{nm}$ to $105 \mathrm{~nm}$, both TE and TM polarized light has a high transmission efficiency of $>90 \%$ and a polarization extinction ratio of $>12 \mathrm{~dB}$.

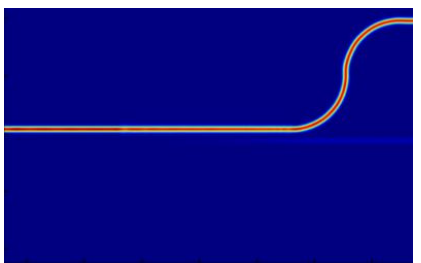

(a)

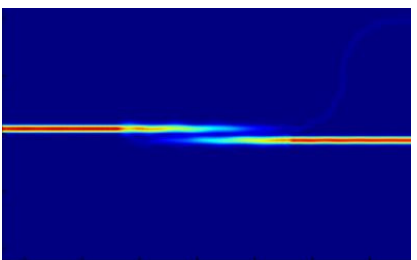

(b)
Fig. 4. (a) TE and (b) TM polarized light propagation along the optimized PBS.

Taking a comprehensive consideration on high transmission efficiency, high polarization extinction ratio, low crosstalk, and fabrication feasibility, we select the side strip width, the slot width, and the coupling length of $200 \mathrm{~nm}, 100 \mathrm{~nm}$, and $15 \mu \mathrm{m}$, respectively, as the optimized parameters of the vertical-dual-slot waveguides based PBS in the $400 \mathrm{~nm}$ thick $4 \mathrm{H}-\mathrm{SiC}$ integrated platform. The simulated TE and TM polarized light propagation at $1550 \mathrm{~nm}$ in the optimized PBS is shown in Fig.4. As can be seen, the TE polarized light is directly guided in the upper waveguide, while the TM polarized light is coupled into the adjacent waveguide, indicating that the proposed structure works effectively. The transmission efficiency and the polarization extinction ratio versus the wavelength are then simulated and plotted in Fig.5. Within the wavelength range between $1510 \mathrm{~nm}$ and $1580 \mathrm{~nm}$, both TE and TM polarized light has a high transmission efficiency of $>90$ $\%$, equivalent to an insertion loss of $<0.46 \mathrm{~dB}$, with a corresponding polarization extinction ratio $>12.5 \mathrm{~dB}$.

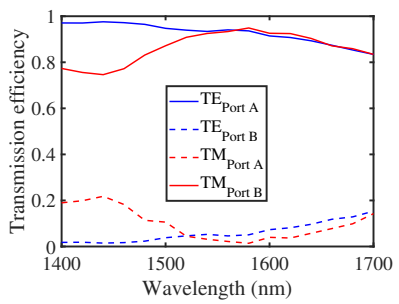

(a)

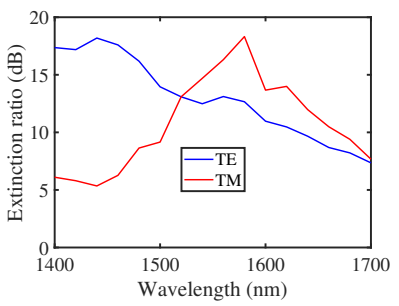

(b)
Fig. 5. The simulated results of (a) transmission efficiency and (b) polarization extinction ratio as a function of wavelength.

\section{FABRICATION}

After optimizing the PBS parameters, we fabricate the device on the $4 \mathrm{H}-\mathrm{SiCOI}$ chip. We fabricate the $4 \mathrm{H}-\mathrm{SiCOI}$ chip through the ion-cut method, shown in Fig. 6(a).

First, a high-purity semi-insulating $4 \mathrm{H}-\mathrm{SiC}$ wafer is implanted with dihydrogen cations, which is on-axis cut with the extraordinary optical axis parallel to the $\mathrm{c}$ axis of the crystal and perpendicular to the wafer plane, with energy of $340 \mathrm{keV}$ and dose of $6 \times 10^{16} \mathrm{~cm}^{-2}$ at $200^{\circ} \mathrm{C}$ to generate a damaged layer underneath the wafer surface at a depth of $1.26 \mu \mathrm{m}$. Second, a silicon wafer, as the substrate, is thermally oxidized to form $2.42 \mu \mathrm{m}$ thick $\mathrm{SiO}_{2}$, as a buried oxide layer. Third, the two wafers are cleaned through a reverse Radio Corporation of America (RCA) cleaning process, and are bonded via hydrophilic 

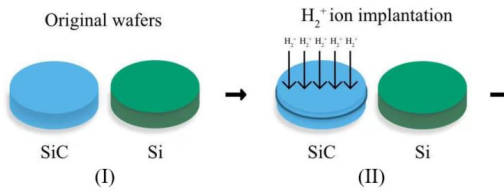

Oxidation and HF dipping

Annealing and splitting

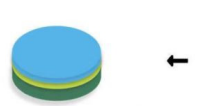

(VI)

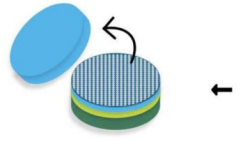

(V)

(a)
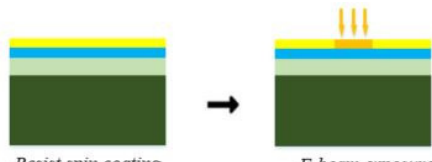

E-beam exposure

(I) Define pattern on the resist

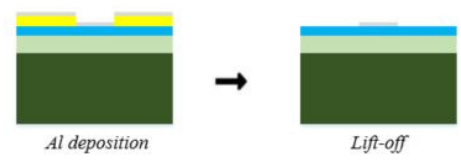

(II) Define pattern on the hard mask

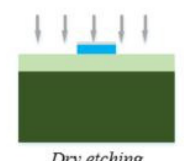

Dry etching

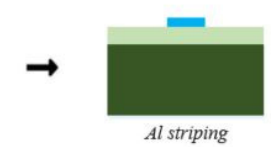

Al striping

(III) Define pattern on the $\mathrm{SiC}$ layer

(b)

Fig. 6. (a) Process flow of the $4 \mathrm{H}-\mathrm{SiCOI}$ stack fabrication. (b) Process flow of the photonic device fabrication. bonding. Fourth, the bonded wafers are annealed at $800^{\circ} \mathrm{C}$ for 6 hours to split the $4 \mathrm{H}-\mathrm{SiC}$ wafer, and a thin $4 \mathrm{H}-\mathrm{SiC}$ film remains on the $\mathrm{Si}$ $\mathrm{SiO}_{2}$ substrate to form the $4 \mathrm{H}-\mathrm{SiCOI}$ stack. Fifth, the thickness of the $4 \mathrm{H}-\mathrm{SiC}$ thin film is reduced down to $\sim 400 \mathrm{~nm}$ via thermal oxidation and HF dipping. Within a $1 \mathrm{~cm}^{2} 4 \mathrm{H}-\mathrm{SiCOI}$ chip, the thickness of the $4 \mathrm{H}-\mathrm{SiC}$ thin film is $404 \pm 1 \mathrm{~nm}$, and the surface roughness is $1.2 \pm 0.3$ $\mathrm{nm}$, indicating a good uniformity and smoothness. A scanning electron microscope (SEM) image of the cross section of the 4H-SiCOI stack is shown in Fig.7(a). An atomic force microscopy (AFM) image of the chip surface profile is shown in Fig.7(b).

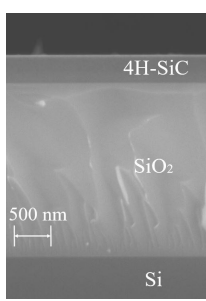

(a)

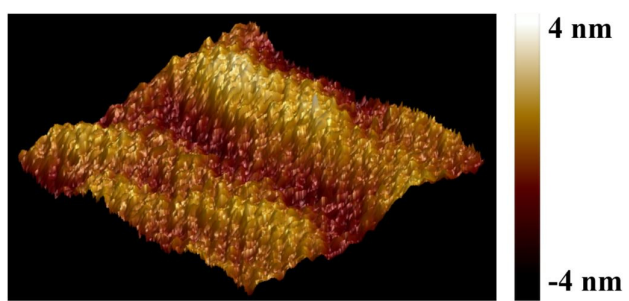

(b)
Fig. 7. (a) SEM image of the $4 \mathrm{H}-\mathrm{SiCOI}$ stack. (b) AFM image of the $4 \mathrm{H}-\mathrm{SiCOI}$ surface topography within an area of $1 \times 1 \mu \mathrm{m}^{2}$.

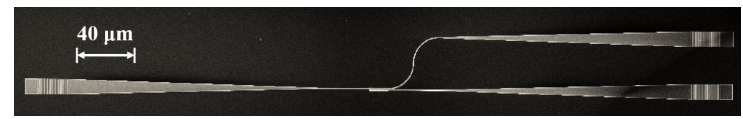

(a)

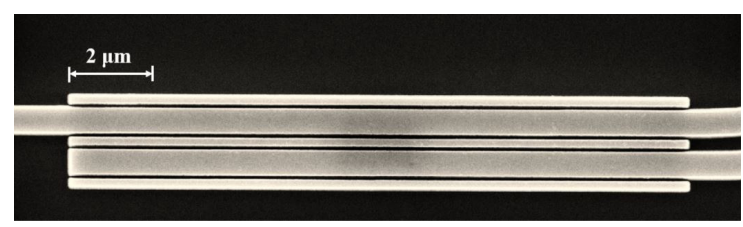

(b)

Fig. 8. (a) SEM image of the fabricated testing device. (b) Zoom-in SEM image of the fabricated vertical-dual-slot waveguides based PBS.

After the $4 \mathrm{H}-\mathrm{SiCOI}$ chip preparation, we fabricate the device on the chip, shown in Fig. 6(b). First, the pattern is defined on the positive electron-beam (e-beam) resist, AR-P 6200.09, through the e-beam writer (JEOL JBX-9500FSZ). Second, the pattern is transferred to an aluminum metal mask through the e-beam evaporation and a lift-off process. Finally, the pattern is transferred to the $4 \mathrm{H}-\mathrm{SiC}$ thin film by inductively coupled plasmon reactive ion etching. The SEM image of the fabricated device is shown in Fig.8(a). At every input and the output port of the beam splitters, polarization-insensitive grating couplers are connected for the device performance characterization [9]. A zoom-in SEM image of the vertical-dual-slot waveguide based PBS is shown in Fig.8(b).

\section{CHARACTERIZATION}

We build the measurement setup, shown in Fig.9, to test the device. A tunable continuous-wave laser source (ANDO AQ4321D) launches light into a polarization controller, to adjust the polarization to quasiTE or quasi-TM mode. The polarized light is coupled in and out of the device with a pair of cleaved fibers, mounted on the fiber holders. 


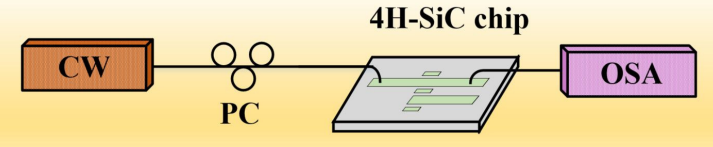

Fig. 9. Measurement setup schematic, $\mathrm{CW}$ : tunable continuous-wave laser, PC: polarization controller, OSA: optical spectrum analyzer.

The output light is detected by an optical spectrum analyzer (ANDO AQ6317B), which allows synchronized sweep with the tunable laser source to measure the transmission spectrum.

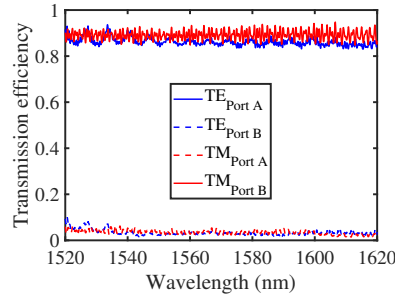

(a)

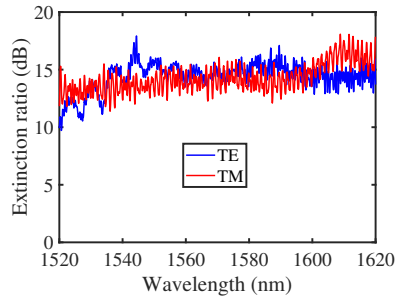

(b)
Fig. 10. The measured results of (a) normalized transmission efficiency and (b) polarization extinction ratio versus wavelength.

The normalized transmission efficiency in the vertical-dual-slot waveguides based PBS of the TE and TM polarized light coupling out of Port A and Port B is plotted in Fig.10(a). The normalized transmission has already excluded the grating transmission for each polarization, and only shows the PBS performance. As can be seen, the transmittance of the TE and TM polarized light in Port A and Port $\mathrm{B}$, respectively, is very high and flat, within the measured wavelength range between $1520 \mathrm{~nm}$ and $1620 \mathrm{~nm}$. The transmission efficiency of TE and TM polarized light is $>85 \%$ and $>89 \%$, equivalent to insertion loss of $<0.71 \mathrm{~dB}$ and $<0.51 \mathrm{~dB}$, respectively. The trend of the transmission efficiency for both TE and TM polarized light versus the wavelength almost match the simulation, but the spectrum has a slight red shift, as there is supposed to be peak at around $1580 \mathrm{~nm}$ according to Fig.5(a). This could be due to the linewidth variation and fabrication imperfections in fabrication processes. Meanwhile, the device also exhibits a low crosstalk, because the transmission efficiency of the TE in Port B and TM polarized light in Port A is very low. The polarization extinction ratio versus the wavelength is plotted in Fig.10(b). As can been seen, the polarization extinction ratio is $>10 \mathrm{~dB}$ within the measured wavelength range for both TE and TM polarized light. Especially, the polarization extinction ratio is $>13 \mathrm{~dB}$, between 1540 $\mathrm{nm}$ and $1620 \mathrm{~nm}$. Compared to the simulation results, the measured polarization extinction ratio of TE polarized light is a little higher, while that of the TM polarized light is a little lower, which is considered to be due to the polarization-dependent propagation loss and coupling loss in the vertical-dual-slot waveguide coupling region, due to the mode distribution and sidewall roughness.

\section{CONCLUSION}

In this paper, a novel vertical-dual-slot waveguides based coupling scheme is proposed for the low-birefringence polarization beam splitting, and is experimentally demonstrated in the $4 \mathrm{H}-\mathrm{SiCOI}$ integrated platform. The polarization beam splitter is very small, with a footprint of $2.2 \times 15 \mu \mathrm{m}^{2}$, which benefits compactness and high-density integration. The measurement results show that it has very low insertion loss of $<0.71 \mathrm{~dB}$ and $<0.51 \mathrm{~dB}$ for the TE and TM polarized light, respectively, within $100 \mathrm{~nm}$ operational bandwidth, and a polarization extinction ratio of $>13 \mathrm{~dB}$ for both TE and TM polarized light, within $80 \mathrm{~nm}$ operational bandwidth, covering most of the $\mathrm{C}$ band and the whole $\mathrm{L}$ band. Our work provides a new solution to split the light beam with low birefringence, and paves ways for the polarization diversity applications in the relatively low-refractive-index material based integrated platforms with high compactness.

Funding. This work was supported by European Union's Horizon 2020 Future and Emerging Technologies Open (SiComb, No.899679) and European Union's Horizon 2020 Research and Innovation (RADIATE, No.824096).

Disclosures. The authors declare no conflicts of interest.

Data availability. Data underlying the results presented in this paper are not publicly available at this time but may be obtained from the authors upon reasonable request.

\section{REFERENCES}

1. D. M. Lukin, C. Dory, M. A. Guidry, K. Y. Yang, S. D. Mishra, R. Trivedi, M. Radulaski, S. Sun, D. Vercruysse, G. H. Ahn et al., "4h-siliconcarbide-on-insulator for integrated quantum and nonlinear photonics," Nat. Photonics 14, 330-334 (2020).

2. S. Castelletto and A. Boretti, "Silicon carbide color centers for quantum applications," J. Physics: Photonics 2, 022001 (2020).

3. X. Shi, W. Fan, A. K. Hansen, M. Chi, A. Yi, X. Ou, K. Rottwitt, and $\mathrm{H}$. Ou, "Thermal behaviors and optical parametric oscillation in 4hsilicon carbide integrated platforms," Adv. Photonics Res. (2021).

4. C. Wang, Z. Fang, A. Yi, B. Yang, Z. Wang, L. Zhou, C. Shen, Y. Zhu, Y. Zhou, R. Bao et al., "High-q microresonators on 4h-silicon-carbideon-insulator platform for nonlinear photonics," Light. Sci. \& Appl. 10, 1-11 (2021).

5. M. A. Guidry, K. Y. Yang, D. M. Lukin, A. Markosyan, J. Yang, M. M. Fejer, and J. Vučković, "Optical parametric oscillation in silicon carbide nanophotonics," Optica 7, 1139-1142 (2020).

6. M. A. Guidry, D. M. Lukin, K. Y. Yang, R. Trivedi, and J. Vučković, "Quantum optics of soliton microcombs," arXiv preprint arXiv:2103.10517 (2021).

7. T. Fan, X. Wu, S. R. Vangapandu, A. H. Hosseinnia, A. A. Eftekhar, and A. Adibi, "Racetrack microresonator based electro-optic phase shifters on a 3c silicon-carbide-on-insulator platform," Opt. Lett. 46, 2135-2138 (2021).

8. S. Castelletto, B. C. Johnson, and A. Boretti, "Quantum effects in silicon carbide hold promise for novel integrated devices and sensors," Adv. Opt. Mater. 1, 609-625 (2013).

9. X. Shi, W. Fan, Y. Lu, A. K. Hansen, M. Chi, A. Yi, X. Ou, K. Rottwitt, and $\mathrm{H}$. Ou, "Polarization and spatial mode dependent four-wave mixing in a 4h-silicon carbide microring resonator," APL Photonics (2021).

10. Y. Tan, H. Wu, and D. Dai, "Silicon-based hybrid (de) multiplexer for wavelength-/polarization-division-multiplexing," J. Light. Technol. 36, 2051-2058 (2018).

11. E. Meyer-Scott, N. Prasannan, C. Eigner, V. Quiring, J. M. Donohue, S. Barkhofen, and C. Silberhorn, "High-performance source of spectrally pure, polarization entangled photon pairs based on hybrid integrated-bulk optics," Opt. express 26, 32475-32490 (2018).

12. J. Wang, D. Liang, Y. Tang, D. Dai, and J. E. Bowers, "Realization of an ultra-short silicon polarization beam splitter with an asymmetrical bent directional coupler," Opt. letters 38, 4-6 (2013).

13. H. Zafar, M. F. Pereira, K. L. Kennedy, and D. H. Anjum, "Fabricationtolerant and cmos-compatible polarization splitter and rotator based on a compact bent-tapered directional coupler," AIP Adv. 10, 125214 (2020).

14. C. Errando-Herranz, S. Das, and K. B. Gylfason, "Suspended polarization beam splitter on silicon-on-insulator," Opt. express 26, 2675-2681 (2018).

15. D. Dai, Z. Wang, J. Peters, and J. E. Bowers, "Compact polarization beam splitter using an asymmetrical mach-zehnder interferometer 
based on silicon-on-insulator waveguides," IEEE Photonics Technol. Lett. 24, 673-675 (2012).

16. C. Li, M. Zhang, J. E. Bowers, and D. Dai, "Ultra-broadband polarization beam splitter with silicon subwavelength-grating waveguides," Opt. letters 45, 2259-2262 (2020).

17. J. Chee, S. Zhu, and G. Lo, "Cmos compatible polarization splitter using hybrid plasmonic waveguide," Opt. Express 20, 25345-25355 (2012).

18. S. Gao, Y. Wang, K. Wang, and E. Skafidas, "Low-loss and broadband $2 \times 2$ polarization beam splitter based on silicon nitride platform," IEEE Photonics Technol. Lett. 28, 1936-1939 (2016).

19. R. Kudalippalliyalil, T. E. Murphy, and K. E. Grutter, "Low-loss and ultrabroadband silicon nitride angled mmi polarization splitter/combiner," Opt. Express 28, 34111-34122 (2020).

20. H. Xu, D. Dai, L. Liu, and Y. Shi, "Proposal for an ultra-broadband polarization beam splitter using an anisotropy-engineered mach-zehnder interferometer on the $\mathrm{x}$-cut lithium-niobate-on-insulator," Opt. express 28, 10899-10908 (2020)

21. J. Zhan, J. Brock, S. Veilleux, and M. Dagenais, "Silicon nitride polarization beam splitter based on polarization-independent mmis and apodized bragg gratings," Opt. Express 29, 14476-14485 (2021).

22. C. Deng, M. Lu, Y. Sun, L. Huang, D. Wang, G. Hu, R. Zhang, B. Yun, and Y. Cui, "Broadband and compact polarization beam splitter in Inoi hetero-anisotropic metamaterials," Opt. Express 29, 11627-11634 (2021).

23. L. Zhang, X. Fu, and L. Yang, "Compact, broadband and low-loss polarization beam splitter on lithium-niobate-on-insulator using a silicon nanowire assisted waveguide," IEEE Photonics J. 12, 1-6 (2020).

24. J. Feng and R. Akimoto, "A three-dimensional silicon nitride polarizing beam splitter," IEEE Photonics Technol. Lett. 26, 706-709 (2014).

25. B. Bhandari, C.-S. Im, O. R. Sapkota, and S.-S. Lee, "Highly efficient broadband silicon nitride polarization beam splitter incorporating serially cascaded asymmetric directional couplers," Opt. Lett. 45, 59745977 (2020).

26. S. Shi and D. W. Prather, "Ultrabroadband electro-optic modulator based on hybrid silicon-polymer dual vertical slot waveguide," Adv. Optoelectronics 2011, 714895 (2011).

27. K. Guo, L. Lin, J. B. Christensen, E. N. Christensen, X. Shi, Y. Ding, K. Rottwitt, and H. Ou, "Broadband wavelength conversion in a silicon vertical-dual-slot waveguide," Opt. Express 25, 32964-32971 (2017).

28. B. E. Little and W.-P. Huang, "Coupled-mode theory for optical waveguides," Prog. In Electromagn. Res. 10, 217-270 (1995). 